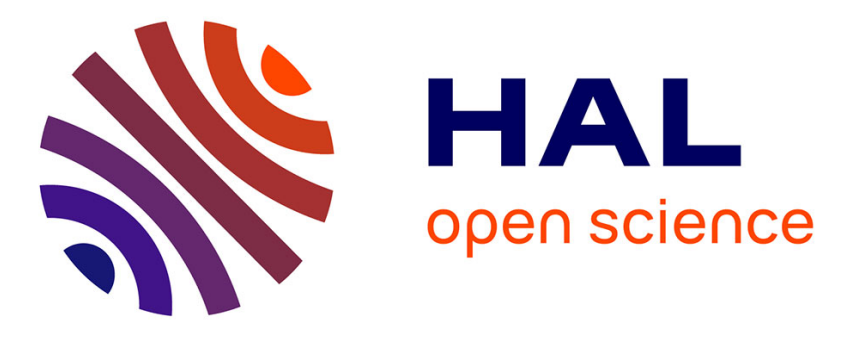

\title{
Resolving the Controversial Existence of Silicene and Germanene Nanosheets Grown on Graphite
}

\author{
Wenbing Peng, Tao Xu, Pascale Diener, Louis Biadala, Maxime Berthe, \\ Xiaodong Pi, Yves Borensztein, Alberto Curcella, Romain Bernard, Geoffroy \\ Prévot, et al.
}

\section{To cite this version:}

Wenbing Peng, Tao Xu, Pascale Diener, Louis Biadala, Maxime Berthe, et al.. Resolving the Controversial Existence of Silicene and Germanene Nanosheets Grown on Graphite. ACS Nano, 2018, 12 (5), pp.4754-4760. 10.1021/acsnano.8b01467 . hal-03037862v2

\section{HAL Id: hal-03037862 \\ https://hal.sorbonne-universite.fr/hal-03037862v2}

Submitted on 8 Dec 2020

HAL is a multi-disciplinary open access archive for the deposit and dissemination of scientific research documents, whether they are published or not. The documents may come from teaching and research institutions in France or abroad, or from public or private research centers.
L'archive ouverte pluridisciplinaire HAL, est destinée au dépôt et à la diffusion de documents scientifiques de niveau recherche, publiés ou non, émanant des établissements d'enseignement et de recherche français ou étrangers, des laboratoires publics ou privés. 


\section{Resolving the Controversial Existence of Silicene and Germanene Nanosheets Grown on Graphite}

Wenbing Peng ${ }^{\dagger, \ddagger}$, Tao $\mathrm{Xu}^{\ddagger}$, , Pascale Diener ${ }^{\ddagger}$, Louis Biadala ${ }^{\ddagger}$, Maxime Berthe ${ }^{\ddagger}$, Xiaodong $\mathrm{Pi}^{\dagger}$, ${ }^{*}$ Yves Borensztein, 'Alberto Curcella, Romain Bernard, "Geoffroy Prévot, ${ }^{\mid *}$ Bruno Grandidier ${ }^{* *}$

${ }^{\dagger}$ State Key Laboratory of silicon Materials, and School of Materials Science and Engineering, Zhejiang University, Hangzhou, Zhejiang 310027, China

† Univ. Lille, CNRS, Centrale Lille, ISEN, Univ. Valenciennes, UMR 8520 - IEMN, F-59000 Lille, France

$\S$ Key Laboratory of Advanced Display and System Application, Shanghai University, 200072, Shanghai, China

' Sorbonne Universités, UPMC Univ Paris 06, CNRS-UMR 7588, Institut des NanoSciences de Paris, F-75005, Paris, France

\section{ABSTRACT}

The highly oriented pyrolytic graphite (HOPG) surface, consisting of a dangling bond-free lattice, is regarded as a potential substrate for van der Waals heteroepitaxy of twodimensional layered materials. In this work, the growth of silicon and germanium on HOPG is investigated with scanning tunneling microscopy by using typical synthesis conditions for silicene and germanene on metal surfaces. At low coverages, the deposition of $\mathrm{Si}$ and $\mathrm{Ge}$ gives rise to tiny and sparse clusters that are surrounded by a honeycomb superstructure. From the detailed analysis of the superstructure, its comparison with the one encountered on the bare and clean HOPG surface and simulations of the electron density, we conclude that the superstructure is caused by charge density modulations in the HOPG surface. At high coverages, we find the formation of clusters, assembled in filamentary patterns, which indicates a Volmer-Weber growth mode instead of a layer-by-layer growth mode. This coverage-dependent study sets the stage for revisiting recent results alleging the synthesis of silicene and germanene on the HOPG surface.

KEYWORDS: silicene, germanene, synthesis, HOPG, scanning tunneling microscopy, electron-density superstructure 
One of the main impetuses for studying graphene stemmed from analogies between the lowenergy physics of the material and relativistic quantum mechanics. ${ }^{1}$ While the honeycomb lattice of graphene gives rise to the formation of Dirac cones, features that do not exist in conventional two-dimensional (2D) semiconductors, the absence of an electronic gap and a weak spin-orbit coupling hampers its extensive use in electronic and spintronic applications. Inspired by this peculiar band structure, a quest has emerged to synthesize other 2D crystals, ${ }^{2}$ since they have raised the hope to find new classes of quantum particles and create more robust quantum computation playgrounds. In particular, it has been shown that other group IV atoms can arrange into 2D atomic crystals with a honeycomb lattice. By analogy with graphene, they have been coined 2D-Xenes. ${ }^{3}$

Nature usually forces 2D crystallites consisting of group IV elements other than carbon to morph into stable three-dimensional forms as their lateral size increases. Only an epitaxial growth on top of a substrate can suppress the deleterious thermal vibrations that are responsible for such transformations. This concept turned out to be successful for the growth of silicene, ${ }^{4-6}$ germanene, ${ }^{7-9}$ and stanene. ${ }^{10,11}$ But, most of the syntheses occurred on metallic substrates, that electronically couple with the 2D-Xenes and hinder the unique physical phenomena expected for such 2D layers. An alternative consists in using substrates, for which a van der Waals interaction is expected. As a result, silicon and germanium have been adsorbed on semimetal, semiconductor and insulating surfaces and there have been claims of the successful formation of $2 \mathrm{D}$ crystallites. ${ }^{12-14}$ But recent works related to the synthesis of silicene on $\mathrm{MoS}_{2}$ and germanene on gold have proved that the interpretation of the experimental results might be different, casting doubt on the previous claims. ${ }^{15,16}$

Similar contradictions exist for the growth of $\mathrm{Si}$ and $\mathrm{Ge}$ on highly oriented pyrolytic graphite (HOPG). Due to their weak interaction with the HOPG surface maintained at room temperature, ${ }^{17} \mathrm{Si}$ and $\mathrm{Ge}$ adatoms could have enough freedom to bond in a honeycomb lattice. Such hypothesis has been recently proposed in Ref. 18 and 19, where honeycomb superstructures were observed and assigned to silicene and germanene islands. But several 
studies had previously reported the formation of clusters instead of $2 \mathrm{D}$ sheets, ${ }^{22-23}$ indicating an island growth mode, also called Volmer-Weber. As the growth of silicene and germanene has been shown to occur in a narrow temperature window with a very small flux of $\mathrm{Si}$ or $\mathrm{Ge}$ atoms, ${ }^{8,24,25}$ such specific growth conditions may not have been precisely considered in all studies. Moreover, as proposed in Ref. 18 and 19, both monolayers and 3D islands could be present on the substrate surface. Therefore, we have studied the adsorption and growth of silicon and germanium on HOPG with scanning tunnelling microscopy (STM) in ultrahigh vacuum (UHV) in a more comprehensive manner.

First, we show that a $(\sqrt{3} \times \sqrt{3}) R 30^{\circ}$ honeycomb superstructure, induced by the presence of defects such as step edges, can be found on the pristine HOPG surface, consistent with the literature. ${ }^{26,27}$ Then we reproduce the experimental conditions described in Ref. 18 for the growth of silicon on the HOPG surface. It is observed that areas with a similar honeycomb structure in the vicinity of Si clusters and step edges occur. As a third step, we have investigated the growth of germanium considering different growth conditions and coverages up to one monolayer (ML). At small Ge coverages, Ge clusters are formed and are also surrounded by a honeycomb structure. Interestingly, for either Si or Ge, the analysis of the honeycomb superstructure reveals the same $(\sqrt{3} \times \sqrt{3}) R 30^{\circ}$ structure with respect to the triangular lattice of graphite. This is in marked contrast with the lattice structures expected for silicene and germanene on HOPG. ${ }^{18,19,28}$ At high Ge coverages, the growth gives rise to filamentary patterns of Ge clusters. Similar results are also obtained for high Si coverages. Based on the limited extent of the honeycomb structure at small coverages and the growth of clusters at higher coverages, we conclude that no 2D growth mode takes place, regardless of the growth conditions. This result questions the putative epitaxial growth of germanene and silicene on the HOPG surface. Conversely, we show that the $(\sqrt{ } 3 \times \sqrt{ } 3) R 30^{\circ}$ honeycomb superstructure is well reproduced by taking into account the interferences resulting from electron wave functions scattered by defects on the HOPG surface. 


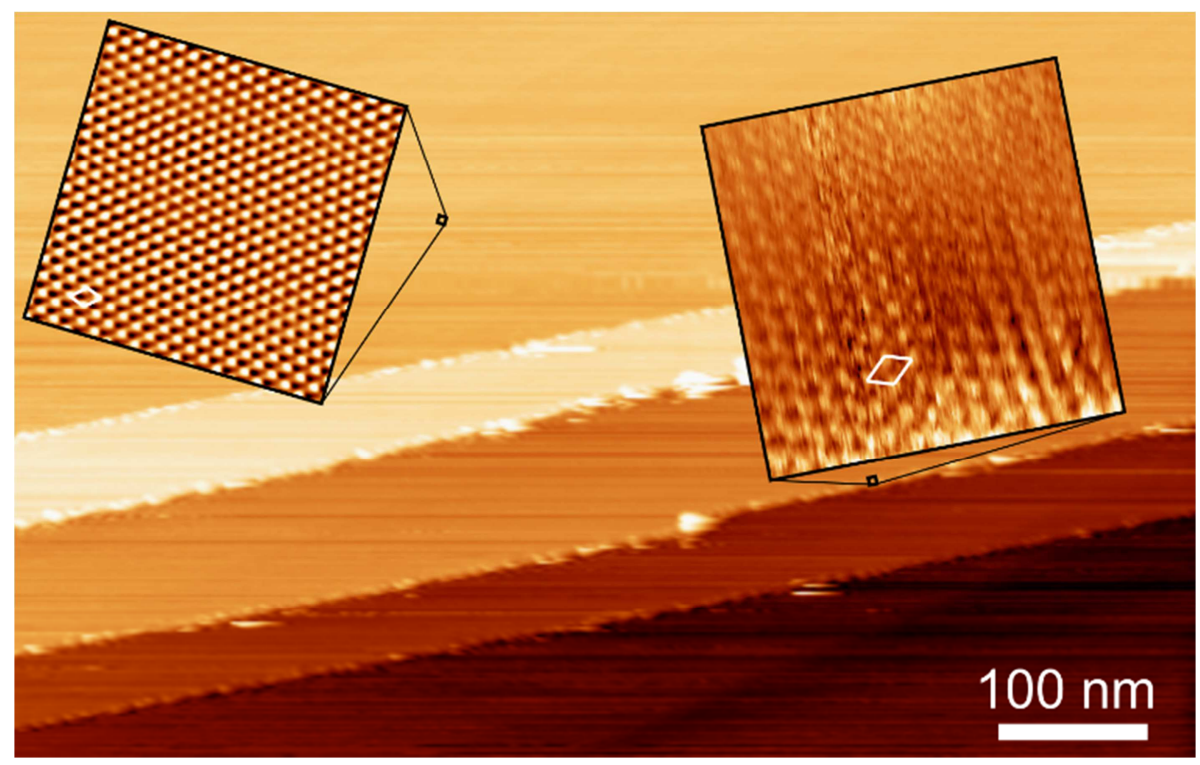

Figure 1. Large scale STM image of the clean HOPG surface obtained at $77 \mathrm{~K}$. Tunneling conditions: $\mathrm{V}_{\text {sample }}=-1$ $\mathrm{V}, \mathrm{I}_{\text {setpoint }}=50 \mathrm{pA}$. Inset: (left) Atomic-resolved STM image revealing the triangular lattice of pristine graphite. Tunneling conditions: $\mathrm{V}_{\text {sample }}=-0.5 \mathrm{~V}, \mathrm{I}_{\text {setpoint }}=100 \mathrm{pA}$. Image size: $4.8 \times 4.8 \mathrm{~nm}^{2}$. (right) High-resolution STM image of a step edge on the HOPG surface. Tunneling conditions: $\mathrm{V}_{\text {sample }}=-0.2 \mathrm{~V}, \mathrm{I}_{\text {setpoint }}=10 \mathrm{pA}$. Image size: 6.3 $x 6.3 \mathrm{~nm}^{2}$. The unit cells for both lattices are superimposed to the STM images.

\section{RESULTS AND DISCUSSION}

Figure 1 shows a typical STM image of the bare HOPG surface, which consists of atomically flat terraces and step edges. Far away from the step edges, the atomicallyresolved image reveals the triangular lattice of graphite with a lattice constant of $2.46 \AA$, which matches the second-nearest neighbour distance of carbon atoms (left inset of Figure 1). This triangular lattice is in agreement with the sub-lattice asymmetry expected for Bernal stacked graphite, for which the three atoms belonging to the first sub-lattice of the surface layer are stacked above the same three atoms of the second layer, while the other three atoms of the surface layer are above the hollow sites of the carbon hexagon of the second layer. ${ }^{29}$ By contrast, in the vicinity of step edges, where the edge of the terrace appears fuzzy, the triangular lattice is no longer visible. Instead a $(\sqrt{3} \times \sqrt{3}) R 30^{\circ}$ superstructure appears on the upper terrace (right inset of Figure 1). Based on the lattice constant of the triangular lattice, we measure a periodicity of $4.3 \pm 0.1 \AA$, consistent with a lattice constant of $\sqrt{ } 3 \times 2.46 \AA=4.26 \AA$. This superstructure agrees with the same superstructure usually 
imaged at the edge of the upper terrace and that extends over $30-40 \AA$ from the edge on a HOPG surface. ${ }^{26,27}$ It corresponds to the interference between normal and scattered electron waves.
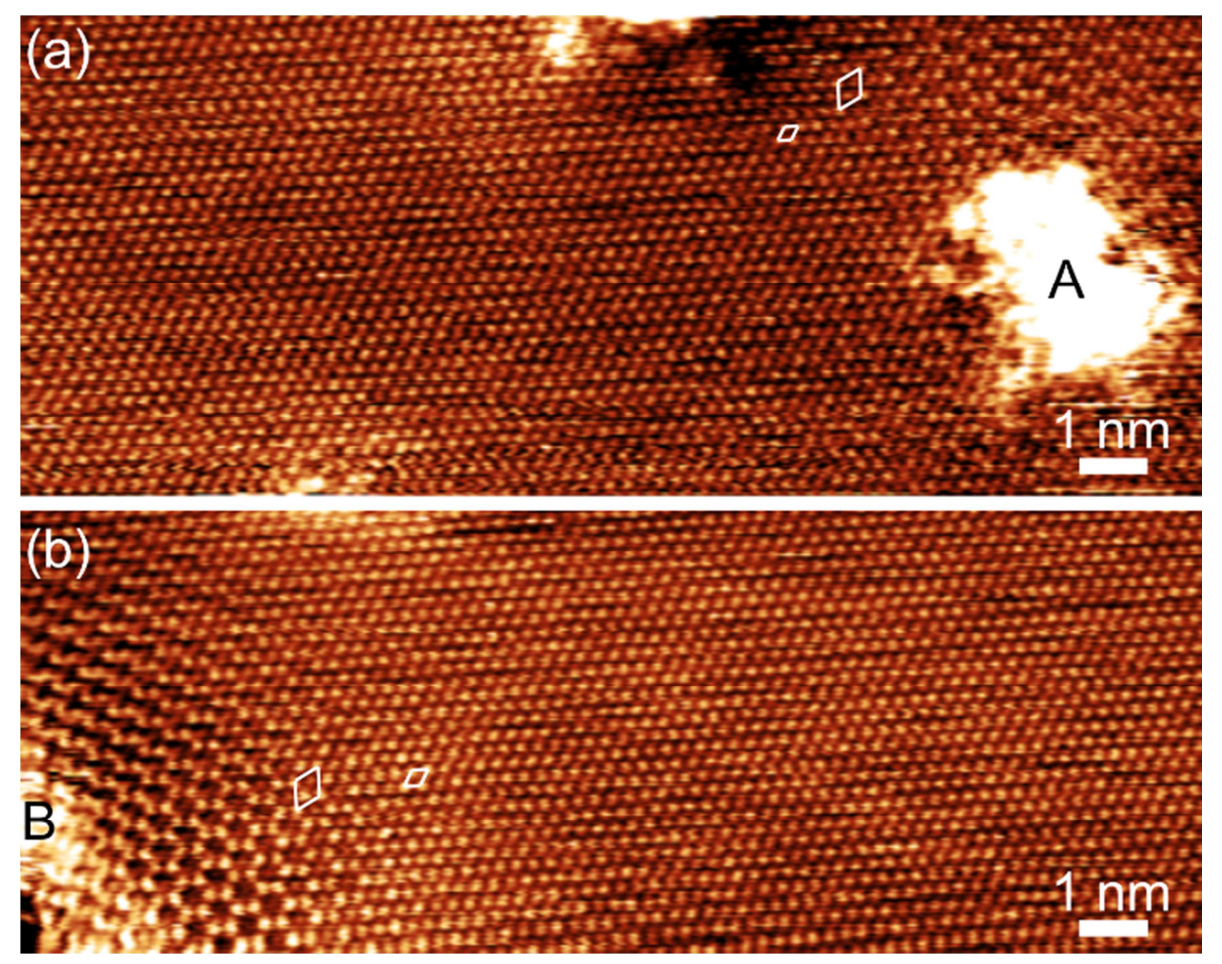

Figure 2. Room temperature STM observation of HOPG after deposition of about $0.2 \mathrm{ML}$ of Si. Two Si clusters discussed in the main text are labelled A, B. The unit cell of HOPG and the one of a $(\sqrt{3} \mathrm{x} \sqrt{3}) R 30^{\circ}$ lattice are superimposed to the STM images. Tunneling conditions: $V_{\text {sample }}=0.20 \mathrm{~V}, \mathrm{I}_{\text {setpoint }}=50 \mathrm{pA}$.

After the deposition of Si atoms on the HOPG surface maintained at room temperature, bright protrusions, similar to the ones shown in Ref. 18, were found on the surface (Figure 2 and Figure 2-SI). We attribute these protrusions to small Si clusters, as demonstrated in Ref. 22. The apparent height of the clusters $A$ and $B$ is about $4 \AA$. We note that cluster $B$ grew along a substrate step edge. While the major part of the atomically flat surface scanned between the clusters shows the triangular lattice of the HOPG surface, the areas in the vicinity of the clusters exhibit different reconstruction patterns. 

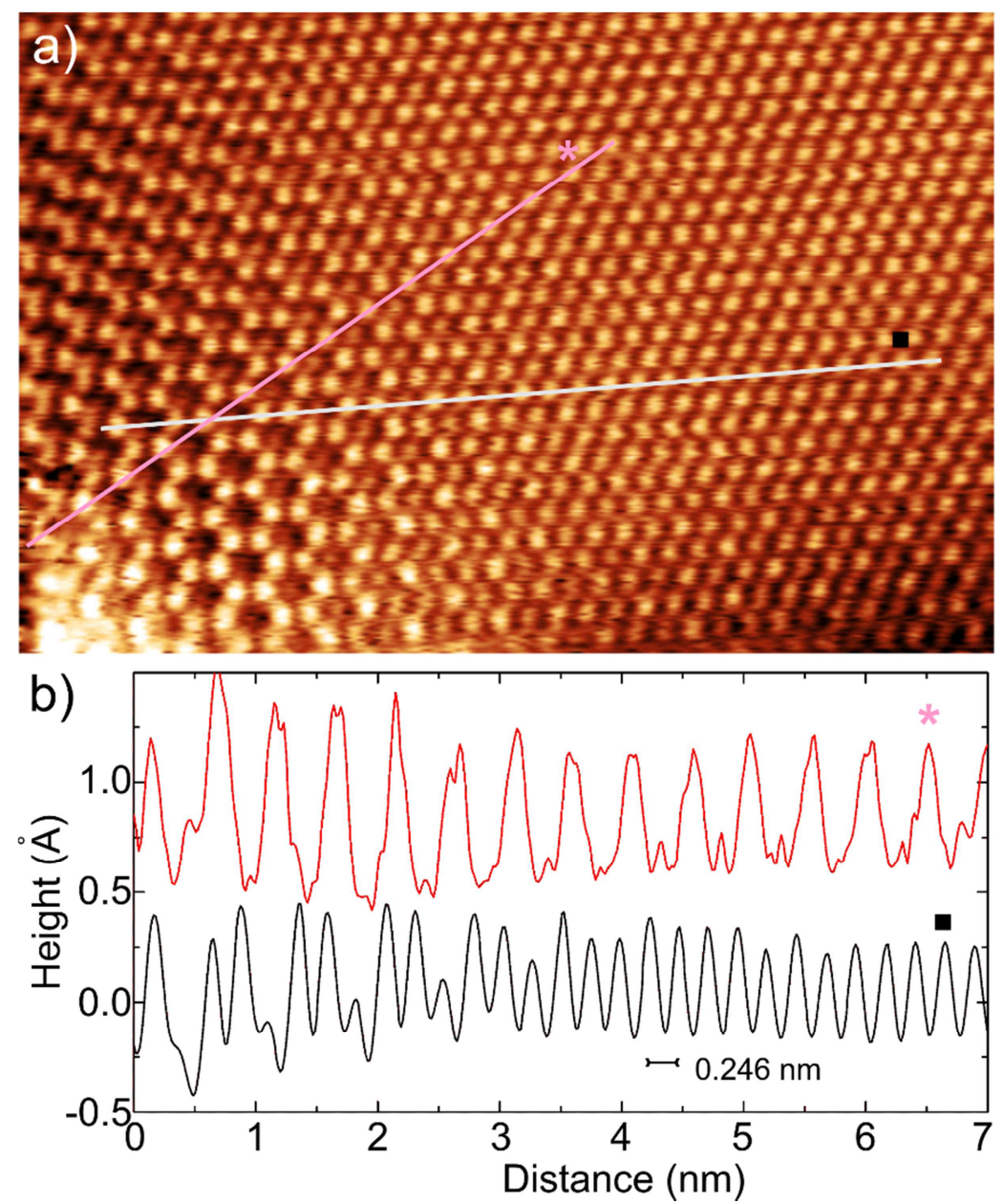

Figure 3. (a) High magnification STM view of the left down part of Figure 2. (b) Height profile along the white and pink segments shown in (a). The STM has been calibrated with respect to the unreconstructed HOPG. Tunneling conditions: $\mathrm{V}_{\text {sample }}=0.20 \mathrm{~V}, \mathrm{I}_{\text {setpoint }}=50 \mathrm{pA}$.

Figure 3 is a high resolution image of the bottom left part of Figure $2 b$. After accurate calibration of the STM image with respect to the unreconstructed HOPG, the lattice constant of the honeycomb superstructure is measured equal to $4.25 \pm 0.05 \AA$, which is identical to the lattice constant of the $(\sqrt{3} \times \sqrt{3}) R 30^{\circ}$ superstructure of the clean and bare HOPG surface. It is worth noticing that, in Ref. 18, the measured lattice constant of the honeycomb areas is similar $(4.1 \pm 0.2 \AA)$ to the one measured here. We must stress that in both cases, the measured lattice constant of the $(\sqrt{3} \mathrm{x} \sqrt{3}) R 30^{\circ}$ honeycomb superstructure disagrees with the one expected for silicene. For a $30^{\circ}$ rotation between silicene and HOPG, ab-initio simulations performed in Ref. 18Erreur ! Signet non défini. predict that a weak interaction leads to a silicene lattice constant equal to $3.79 \AA$, close to the one of free-standing silicene 
(3.83 $\AA$ ). It is $11 \%$ smaller than the measured value (experimental uncertainty $<2 \%$ ), which is not compatible with the observed $(\sqrt{3} \mathrm{x} \sqrt{3}) R 30^{\circ}$ superstructure cell. Moreover, in the close case of silicene on graphene, Cai et $a .^{28}$ predicted that multiple phases of single crystalline silicene with different orientations relative to the graphene substrate could coexist at room temperature. A large number of simulated configurations have been found to have nearly the same free energy, the most stable one corresponding to the coincidence between a $(\sqrt{21} \times \sqrt{ } 21) R 10.9^{\circ}$ silicene supercell and a $(2 \sqrt{ } 13 \times 2 \sqrt{ } 13) R 13.9^{\circ}$ graphene supercell. Whereas such a variety of reconstructions are observed for $\mathrm{Si} / \mathrm{Ag}(111),{ }^{24,25}$ all reconstructions observed on HOPG after $\mathrm{Si}$ evaporation correspond to $(\sqrt{3} \times \sqrt{3}) R 3$ superstructures. Therefore, this superstructure is unlikely to be a silicene nanosheet weakly interacting with HOPG.

Additionally, it is also clear in Figure 3 that there is a continuous modification from the unreconstructed areas of graphite displaying the typical triangular arrangement (right side) to the honeycomb superstructure area close to the cluster (left side). This change takes along with a slight increase of the corrugation, as seen in the height profiles of Figure 3b, acquired along similar directions than the ones shown in Ref. 18. If the latter area were caused by silicene, we would not expect such a graduate change from the unreconstructed area to the honeycomb superstructure, extending over a distance of $30 \AA$. Instead a sharp transition would rather be obtained. Interestingly, an atom is located at the centre of the honeycomb. Its contrast progressively fades out from the unreconstructed region to the honeycomb superstructure one. This central atom is not expected if the superstructure consists of a silicene nanosheet located $3.3 \AA$ above the graphite surface, as proposed from calculation in Ref 18. In order to better evidence these observations, Figure $3 \mathrm{~b}$ displays the measured profile along the white line indicated in Figure 3a, which shows the variation of the apparent height of this central atom. Focussing on the left part of the profile, that corresponds to the superstructure, it appears that two neighbouring atoms do not have exactly the same height, suggesting a buckling effect, similar to the one described in Figure $3 \mathrm{c}$ of Ref. 18. However, the positions of the up and down atoms of the honeycomb superstructure match perfectly the 
positions of the carbon atoms of the unreconstructed graphite, questioning the assignment of the buckling effect to the presence of silicene in Ref. 18.
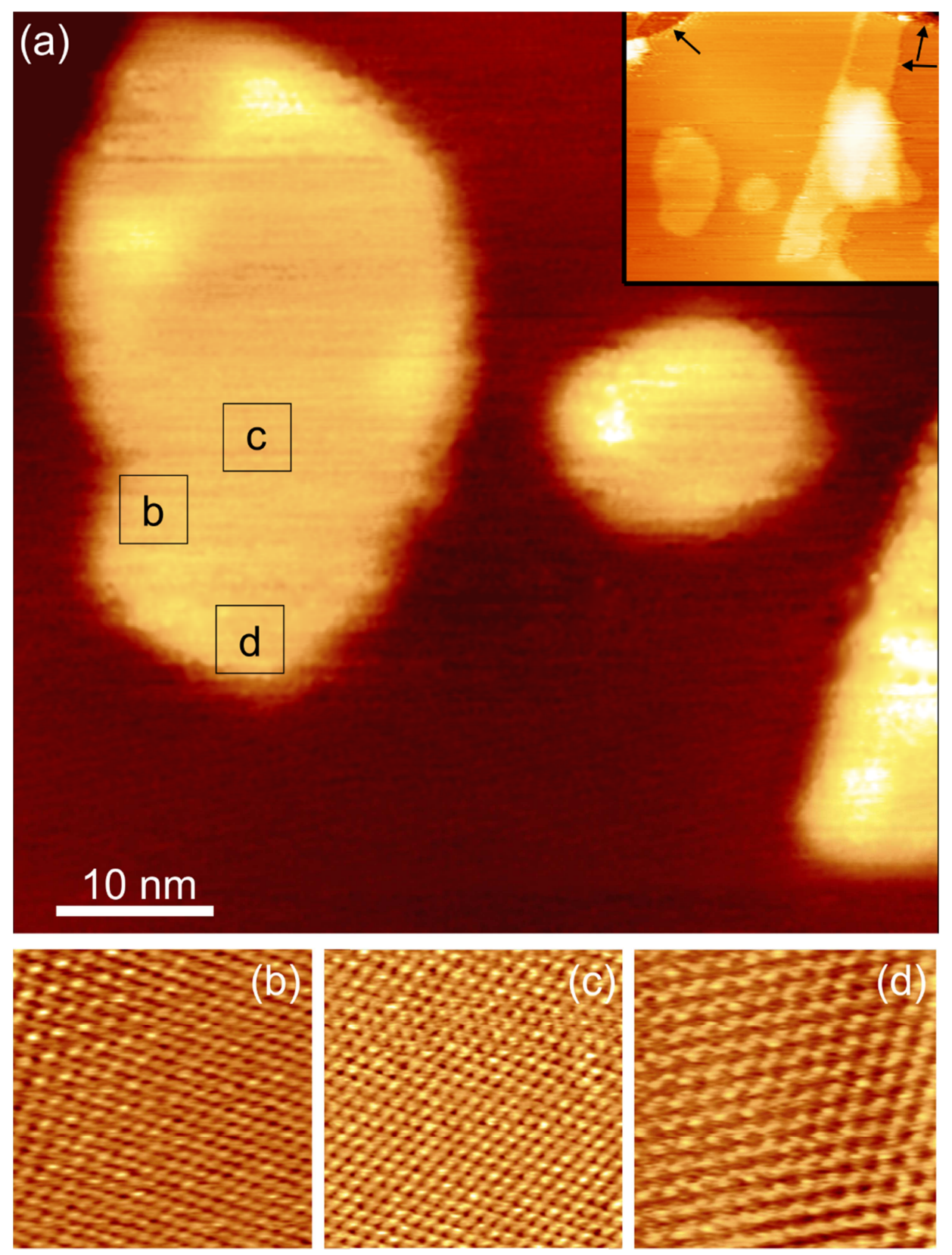

Figure 4. (a) STM image of the HOPG surface after the deposition of $0.1 \mathrm{ML}$ of $\mathrm{Ge}$ at $85^{\circ} \mathrm{C}$ and a subsequent annealing at $150^{\circ} \mathrm{C}$. Inset: STM image of the same area at a larger scale where arrows highlight steps of the HOPG surface that were used to calibrate the vertical axis of the scanner. Tunneling conditions: $V_{\text {bias }}=+1 \mathrm{~V}$, $I_{\text {setpoint }}=5 \mathrm{pA}$. The squares in (a) indicate the position where the atomically resolved STM images in (b), (c) and d) were obtained. Tunneling conditions: $\mathrm{V}_{\text {bias }}=150 \mathrm{mV}(\mathrm{b}), 100 \mathrm{mV}$ (c), $150 \mathrm{mV}(\mathrm{d}), \mathrm{I}_{\text {setpoint }}=10 \mathrm{pA}$. The images were obtained at $77 \mathrm{~K}$. 
It has recently been demonstrated that germanene can be grown on $\mathrm{Al}(111)$ when this crystal is maintained at $85^{\circ} \mathrm{C}$ during Ge evaporation. ${ }^{8}$ Hence, we first study the growth of $\mathrm{Ge}$ on HOPG heated at $85^{\circ} \mathrm{C}$. After evaporation of $\mathrm{Ge}$ at a coverage of $0.1 \mathrm{ML}$, the surface is covered with small bright protrusions. However, the STM images obtained at 77K are usually fuzzy, as shown in the Figure 1a-SI. Although the protrusions are randomly found on the surface, they show a higher probability to reside close to step edges. As they are not seen prior to the Ge evaporation, we attribute them to $\mathrm{Ge}$ adsorbates. In the vicinity of areas corresponding to step edges, where the diffusion of the Ge protrusions is much reduced, superstructures with a honeycomb lattice could be observed. But the instability of the tunnelling junction, certainly due to the diffusion of $\mathrm{Ge}$ atoms or tiny $\mathrm{Ge}$ clusters under the STM tip, does not allow for accurately determining the origin of the pattern. Therefore, the sample is further annealed at $150^{\circ} \mathrm{C}$ for 2 hours.

After the annealing, sparse islands are found on the atomically flat terraces of the HOPG surface consistent with those shown in Ref. 19. Such islands with limited size and rounded boundaries are in contrast with the extended terraces obtained after the exfoliation of the top HOPG layer. ${ }^{30}$ Therefore we can reasonably wonder if these islands could be due to stable germanene nanosheets. From the height calibration obtained from the different terraces observed in the inset of Figure $4 \mathrm{a}$, we find an apparent island height of $3.3 \pm 0.1 \AA$, which is close to the predicted separation of $3.28 \AA$ between germanene and HOPG. ${ }^{19}$ Nevertheless, this value corresponds to the interlayer spacing of graphite.

In order to disclose the nature of the island, high-resolution STM images are acquired on the largest island seen in Figure 4a. Interestingly, superstructures are observed at the boundaries of the islands (Figure $4 \mathrm{~b}$ and $\mathrm{d}$ ). In particular, Figure $4 \mathrm{~d}$ shows a honeycomb structure with a lattice constant of $4.3 \pm 0.1 \AA$, in very good agreement with the one reported in Ref. 19 and consistent with the ones predicted for a hypothetical germanene/graphene bilayer. ${ }^{28}$ However, the geometry of the observed pattern matches the one measured in Figure 1 and $3 b$, corresponding to a $(\sqrt{3} \mathrm{x} \sqrt{3}) R 30^{\circ}$ honeycomb structure. When the tip scans 
a few nanometres away from the island edge (Figure 4c), the structure changes to show the typical triangular lattice of graphite with a periodicity of $2.46 \pm 0.10 \AA$. The latter is seen everywhere in the centre of the islands. Again, as shown in Figure 4b, the transition between the superstructures (top left) and the triangular lattice (bottom right) is smooth without any significant height variation. Moreover, the observation of the island in Figure 4 a clearly reveals that the top of the island is atomically flat in this region. Therefore, the existence of a triangular lattice with the unit cell of graphite in the major part of the island indicates a graphitic island. Although the adsorption of Ge atoms on the surface may be involved in the observation of these islands, since they were not visible prior to the Ge deposition (see section 3 of the supporting information), the $(\sqrt{3} \times \sqrt{3}) R 30^{\circ}$ honeycomb structure cannot be related to the epitaxial growth of a germanene nanosheet.

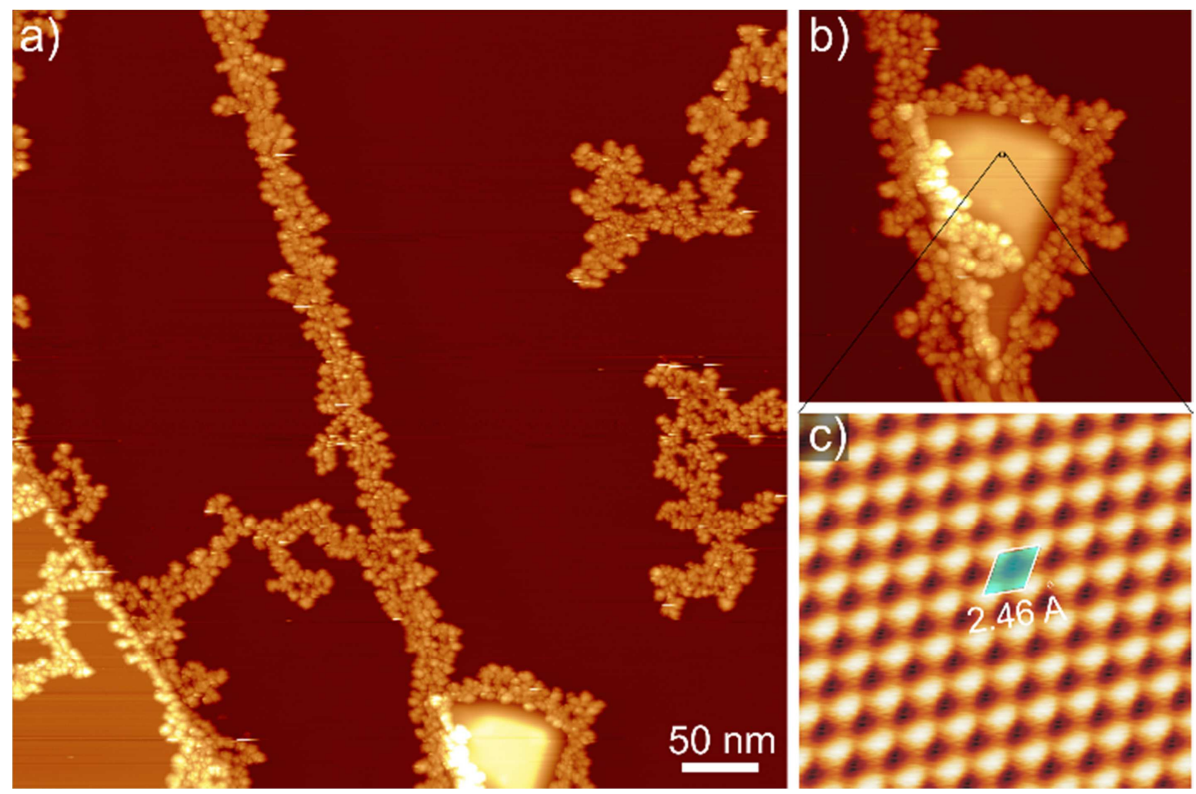

Figure 5. (a) Large scale STM image of the HOPG surface after the deposition of $1.0 \mathrm{ML}$ of $\mathrm{Ge}$ at $120^{\circ} \mathrm{C}$. (b) Zoom on the small island seen at the bottom of (a). (c) Atomic-resolved STM image obtained in the center of the island showing the triangular lattice of the HOPG surface. Tunneling conditions: (a) and (b) $\mathrm{V}_{\text {bias }}=+5 \mathrm{~V}, \mathrm{I}_{\text {setpoint }}=$ $5 \mathrm{pA}$, (c) $\mathrm{V}_{\text {bias }}=+0.15 \mathrm{~V}, \mathrm{I}_{\text {setpoint }}=30 \mathrm{pA}$. The images were obtained at $77 \mathrm{~K}$.

At high coverages, keeping the same temperature range of the typical synthesis of germanene on the $\mathrm{Al}(111)$ surface, ${ }^{8,31}$ the STM observation of the HOPG surface reveals the presence of small flat islands and clusters. As shown in Figure 5a, the clusters decorate the 
step edges of the HOPG surface, giving rise to filamentary patterns in the middle of atomically flat terraces. Their mean size is about $2 \mathrm{~nm}$ (see the height profile in Figure 3b$\mathrm{SI})$. They are much more stable than the Ge protrusions obtained at a coverage of $0.1 \mathrm{ML}$. Since the HOPG surface has a triangular lattice away from the area filled with clusters, we focus on the analysis of the atomic lattice of small cluster-free flat islands (Figure 5b), looking for germanene nanosheets. However, from the atomically resolved STM images (Figure 5c), we unambiguously show that these islands have the triangular lattice of graphite. This observation indicates that the synthesis of $2 \mathrm{D}$ islands of germanene does not take along with the nucleation of clusters at this particular temperature of $120^{\circ} \mathrm{C}$ for the HOPG surface and a low flux during the deposition of Ge. Importantly, in the case of $\mathrm{Ge}$ deposition on $\mathrm{Al}(111)$ surfaces, similar growth conditions lead to a full coverage of the Al terraces by germanene nanosheets (see Figure 5-SI).
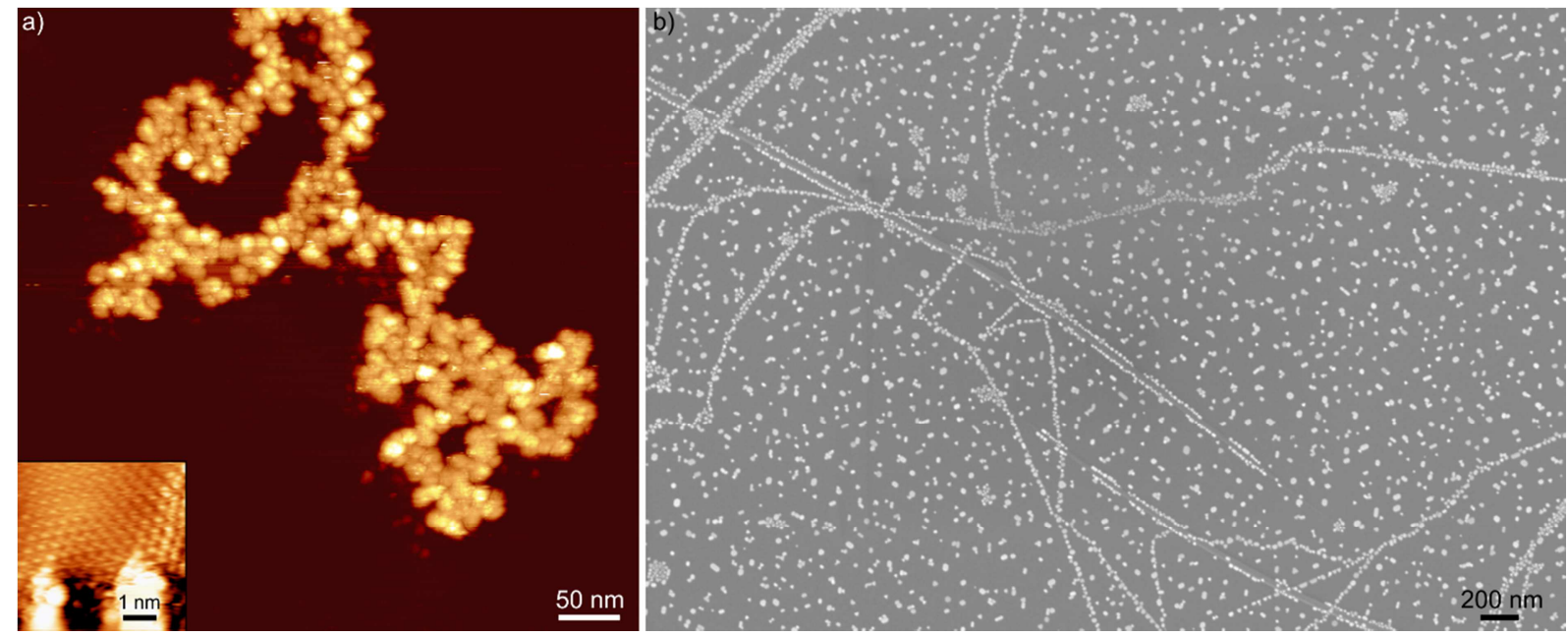

Figure 6. a) STM image of the HOPG surface after the deposition of $0.6 \mathrm{ML}$ of $\mathrm{Ge}$ at room temperature. Tunneling conditions: $\mathrm{V}_{\text {sample }}=+5.0 \mathrm{~V}, \mathrm{I}_{\text {setpoint }}=10 \mathrm{pA}$. Inset : Area showing the honeycomb structure in the vicinity of isolated Ge clusters. Tunneling conditions: $V_{\text {sample }}=200 \mathrm{mV}$, Isetpoint $=8 p A$. The images were obtained at $77 \mathrm{~K}$. b) Scanning electron micrograph of the HOPG surface after the deposition of 1.0 ML of Si at room temperature.

Ge and Si were also evaporated at a higher coverage with the HOPG maintained at room temperature. In the STM image of Figure 6a, obtained after the deposition of $0.6 \mathrm{ML}$ of Ge, a filamentary pattern is observed again. It consists of irregular clusters with a mean size of $2 \mathrm{~nm}$ (Figure $3 \mathrm{~d}-\mathrm{SI}$ ). Similarly, in the scanning electron microscope image of Figure 6b, 
acquired after the deposition of $1.0 \mathrm{ML}$ of $\mathrm{Si}$, numerous clusters are seen. Some of them are sprinkled on the atomically flat terraces. But the majority decorates the step edges of the HOPG surface. Due to the very poor reactivity of the HOPG surface, adsorbed $\mathrm{Ge}$ and $\mathrm{Si}$ adatoms can easily diffuse onto this surface and then aggregate to form clusters. The formation of clusters is thus consistent with the results found by previous works, which studied the growth of Ge on the HOPG surface, despite the fact a higher Ge / Si flux was used in the previous studies. ${ }^{21-22}$ Away from these clusters, the analysis of atomically flat areas does not reveal a lattice spacing that is different from the triangular lattice of graphite in regions free of $\mathrm{Ge}$ or $\mathrm{Si}$ islands. Such an observation excludes the formation of germanene and silicene nanosheets on top of the HOPG surface. Only in the surroundings of isolated clusters, superstructures appeared, as shown in the inset of Figure 6a. Again, the area showing the superstructure is limited, which cannot be attributed to the growth of a 2D layered materials.

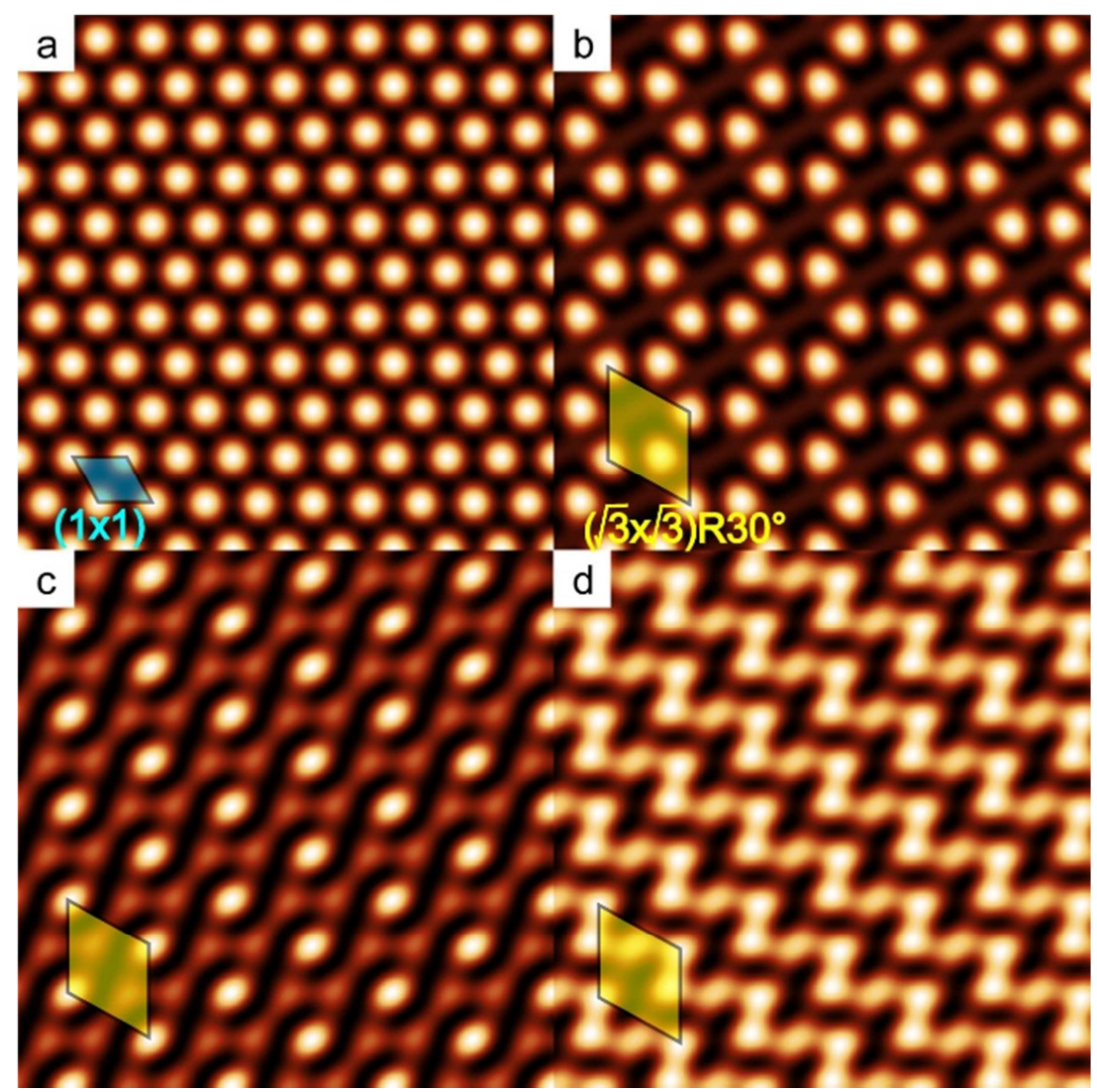

Figure 7. Simulated local density of states at the Fermi level, for various set of $a_{i}$ and $b_{i}$ coefficients. a) $a_{i}=b_{i}=$ 1, b) $a_{1}=a_{2}=0, a_{3}=b_{1}=1$, c) $a_{1}=a_{2}=b_{2}=b_{3}=1, a_{3}=2, b_{1}=0$, d) $a_{1}=b_{2}=1, a_{2}=b_{1}=0, a_{3}=b_{3}=2$. 
With or without the presence of $\mathrm{Si}$ and $\mathrm{Ge}$ on the HOPG surface, most of the superstructures show the same $(\sqrt{ } 3 \mathrm{x} \sqrt{3}) R 30^{\circ}$ honeycomb lattice with respect to the unit cell of pristine graphite. Moreover, such areas are always limited, which can be found in the vicinity of clusters and step edges, that involve a breakdown of the graphite atomic lattice periodicity. The superstructures could thus result from a long-range perturbation of the charge density in HOPG due to interference phenomena between the surface electron waves and scattered waves from defects or clusters. ${ }^{32-34}$ Similar patterns were also observed after the adsorption of other chemical elements (e. g., ozone ${ }^{32}$ and hydrogen ${ }^{35}$ ), particles (e. g., $\mathrm{Pt}^{36,37} \mathrm{Mo}^{38}$ and $\mathrm{Ag}$ clusters ${ }^{39}$ ), and/or the creation of defects induced by $\mathrm{Ar}^{+}-$ bombardment ${ }^{40-42}$ and heating-induced pits. ${ }^{41}$ In particular, honeycomb superstructure images identical to our observations and those in Ref. 18 have been obtained in the case of Pt clusters ${ }^{37}$ and $\mathrm{Ar}^{+}$induced defects. ${ }^{42}$ In all these cases, the charge density modulations decay from the defects along a distance around 20 to $50 \AA$, depending on the type of defects. This is similar to what is observed here and in Ref. 18.

In order to simulate the modulation of the electron density, we have used a wave function interference model similar to the one developed in Ref. 32 and 33, keeping in mind that, at low bias, the STM images reflect the wave functions of the electron states near the Fermi surface. Therefore, we have restricted the wave planes to the six plane waves corresponding to the different $\mathrm{K}$ and $\mathrm{K}^{\prime}$ points of the Brillouin zone at the Fermi energy. The electron density can then be rewritten as:

$$
n(\vec{r})=\psi_{s}^{2}+\psi_{a}^{2}=\left(\sum_{i=1}^{3} a_{i} \cos \left(\vec{K}_{i} \vec{r}\right)\right)^{2}+\left(\sum_{i=1}^{3} b_{i} \sin \left(\vec{K}_{i} \vec{r}\right)\right)^{2}
$$

where $\psi_{s}$ and $\psi_{a}$ are the symmetric and antisymmetric linear combinations of the six plane waves. For an unperturbed surface, one expects all coefficients to be equal, giving rises to the trigonal pattern in Figure $7 \mathrm{a}$, with a $(1 \times 1)$ periodicity. As soon as the coefficients are not all equal, a $(\sqrt{3} 3 \times \sqrt{3}) \mathrm{R} 30^{\circ}$ pattern forms, as shown in Figure $7 b, c$ and $d$. By varying 
the $a_{i}$ and $b_{i}$ coefficients, we are able to reproduce the various patterns observed in the present study and in the previously published results. ${ }^{18,19}$
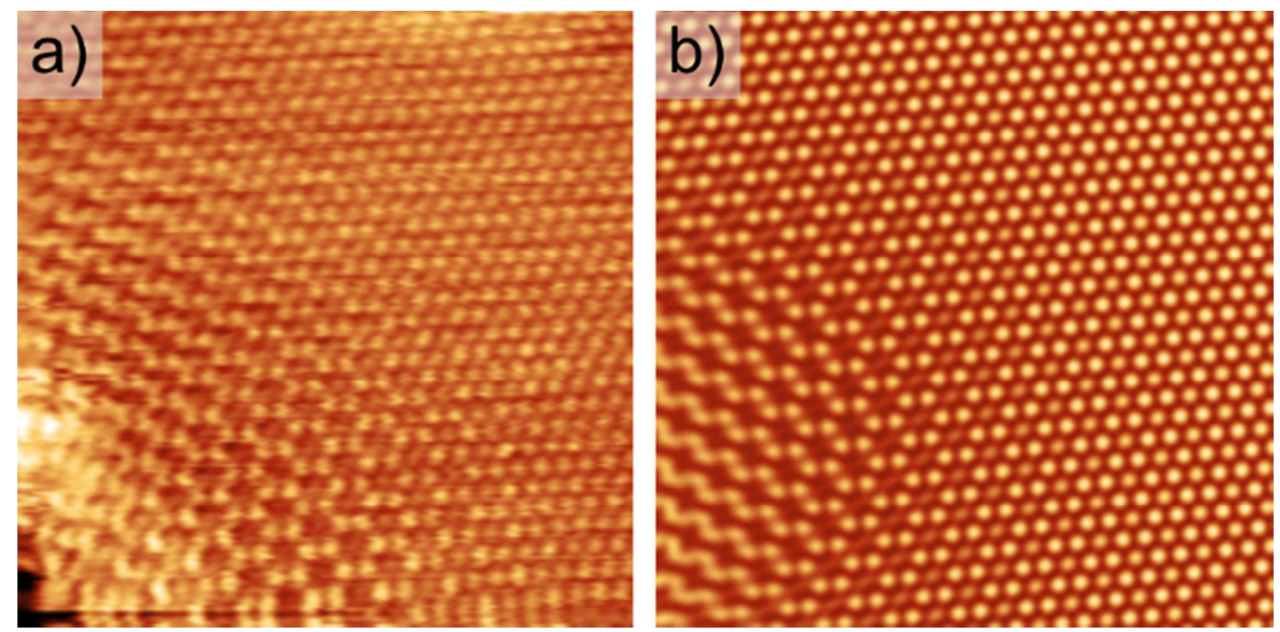

Figure 8. Comparison of (a) the experimental STM image of Figure 3a with (b) a simulated STM image where electronic interferences are considered.

We have also simulated the combined effect of a linear step edge and a silicon island (see Figure 8). For that purpose, we have introduced two perturbation waves, exponentially attenuated from the position of a linear defect mimicking a step edge, and from the position of a circular defect mimicking a Si island. The same attenuation coefficient of $20 \AA$ has been used for both waves. With no attempt of performing a quantitative fit, we reproduce the variations observed in the vicinity of the island that has grown near a step edge. In particular, the transition between a hexagonal pattern and a zigzag chain is well reproduced by the model. Similarly, the transition between the different reconstructions observed in Ref. 18 corresponds to the interference between perturbation waves originating from neighbour silicon islands and does not requires the formation of a silicene nanoribbon between the clusters to account for the existence of a superstructure.

\section{CONCLUSIONS}

In conclusion, the accurate analysis of our STM data demonstrates that the deposition of $\mathrm{Si}$ and Ge on HOPG does not produce silicene and germanene islands for growth conditions 
that are known to lead to the synthesis of silicene and germanene on metal substrates. At low coverage, the well-known honeycomb $(\sqrt{3} \times \sqrt{3}) R 30^{\circ}$ superstructure that is observed at step edges and clusters, reflects surface charge density modulations induced by a breaking of periodicity. At high coverage, the formation of small clusters indicates a Volmer-Weber growth mode which is opposite to van der Waals heteroepitaxy. The current results highlight the challenges of synthesizing $\mathrm{Si}$ and $\mathrm{Ge} 2 \mathrm{D}$ nanosheets on the HOPG surface.

\section{METHODS}

The investigations of silicon and germanium deposition on HOPG were performed in two different ultrahigh vacuum (UHV) systems, on different samples. The preparation of clean and well-ordered HOPG surfaces consisted of two steps: first the top layers of a HOPG substrate were peeled off in air and then the substrate was loaded in UHV to be annealed at $300^{\circ} \mathrm{C}$ for 1 hour. For the deposition of $\mathrm{Si}$ and Ge on the HOPG surface, a monolayer (ML) is referred to the silicene and germanene monolayers that are obtained when the $(3 \times 3)$ silicene unit cell coincides with the $(4 \times 4) A g(111)-(1 \times 1)$ area and the (2x2) germanene unit cell coincides with the $(3 \times 3) \mathrm{Al}(111)-(1 \times 1)$ area. They respectively correspond to $15.7 \times 10^{14} \mathrm{Si}$ atoms.cm $\mathrm{cm}^{-2}$ and $12.6 \times 10^{14} \mathrm{Ge}$ atoms.cm ${ }^{-2}$. Si was evaporated at rates below $0.02 \mathrm{ML} / \mathrm{min}$, using an Omicron Nanotechnology e-beam evaporator and a base pressure of $1.6 \times 10^{-10}$ Torr. The deposition was achieved with the HOPG substrate already fixed on the STM head of a variable-temperature microscope working at room-temperature. The evaporation of $\mathrm{Ge}$ onto the HOPG substrate was performed with a direct-current heating of a $\mathrm{Ge}(001)$ substrate held at $800^{\circ} \mathrm{C}$ and a base pressure of $2.0 \times 10^{-10}$ Torr, yielding small deposition rates of $0.008 \mathrm{ML} / \mathrm{min}$. During the evaporation process, the HOPG substrate was either left at room temperature or heated at temperatures in the range $85 \sim 120^{\circ} \mathrm{C}$ to adopt similar growth conditions as the ones encountered for the synthesis of germanene on $\mathrm{Al}(111) .{ }^{8}$ In the latter case, an $\mathrm{Al}(111)$ crystal was used to ensure that similar growth conditions led to the successful formation of germanene, as shown in Figure S2-SI. After Ge deposition, the 
sample was transferred into the analysis chamber of the UHV system and imaged with a lowtemperature scanning tunnelling microscope (STM) working at $77 \mathrm{~K}$. In all experiments, several W STM tips, electrochemically etched and then heated in UHV, were used to ensure the reproducibility of the experimental findings described below and avoid possible tip artefacts. ${ }^{43}$

\section{ASSOCIATED CONTENT}

The Supporting Information is available free of charge on the ACS Publications website at DOI:

Supplemental STM images and height profiles.

\section{AUTHOR INFORMATION}

Corresponding authors.

*E-mail: bruno.grandidier@isen.iemn.univ-lille1.fr;

geoffroy.prevot@insp.sorbonne universite.fr;

xdpi@zju.edu.cn

\section{ACKNOWLEDGMENTS}

We thank C. Pirri and M. Derivaz for their assistance in the sample preparation. This study is financially supported by the Natural Science Foundation of China (Grant No. 6147409), the French National Research Agency (Germanene project ANR-17-CE09-0021-03), the French state funds managed by the ANR within the Investissements d'Avenir programme LABEX MATISSE (ANR-11-IDEX-0004-02) and EQUIPEX Excelsior (ANR-11-EQPX-0015), the RENATECH network. 


\section{REFERENCES}

(1) Polini, M.; Guinea, F.; Lewenstein, M.; Manoharan, H. C.; Pellegrini, V. Artificial Honeycomb Lattices for Electrons, Atoms and Photons. Nat. Nanotechnol. 2013, 8, 625-633.

(2) Novoselov, K. S.; Jiang, D.; Schedin, F.; Booth, T. J.; Khotkevich, V. V.; Morozov, S. V.; Geim, A. K. Two-Dimensional Atomic Crystals. Proc. Natl. Acad. Sci. 2005, 102, 10451-10453.

(3) Molle, A.; Goldberger, J.; Houssa, M.; Xu, Y.; Zhang, S. C.; Akinwande, D. Buckled TwoDimensional Xene Sheets. Nat. Mater. 2017, 16, 163-169.

(4) Vogt, P.; De Padova, P.; Quaresima, C.; Avila, J.; Frantzeskakis, E.; Asensio, M. C.; Resta, A.; Ealet, B.; Le Lay, G. Silicene: Compelling Experimental Evidence for Graphenelike TwoDimensional Silicon. Phys. Rev. Lett. 2012, 108, 155501.

(5) Feng, B.; Ding, Z.; Meng, S.; Yao, Y.; He, X.; Cheng, P.; Chen, L.; Wu, K. Evidence of Silicene in Honeycomb Structures of Silicon on Ag (111). Nano Lett. 2012, 12, 3507-3511.

(6) Fleurence, A.; Friedlein, R.; Ozaki, T.; Kawai, H.; Wang, Y.; Yamada-Takamura, Y. Experimental Evidence for Epitaxial Silicene on Diboride Thin Films. Phys. Rev. Lett. 2012, 108, 245501.

(7) Dávila, M. E.; Xian, L.; Cahangirov, S.; Rubio, A.; Le Lay, G. Germanene: a Novel TwoDimansional Germanium Allotrope akin to Graphene and Silicene. New J. Phys. 2014, 16, 095002.

(8) Derivaz, M.; Dentel, D.; Stephan, R.; Hanf, M. C.; Mehdaoui, A.; Sonnet, P.; Pirri, C. Continuous Germanene Layer on $\mathrm{Al}(111)$. Nano Lett. 2015, 15, 2510-2516.

(9) Gou, J.; Zhong, Q.; Sheng, S.; Li, W.; Cheng, P.; Li, H.; Chen, L.; Wu, K. Strained Monolayer Germanene with 1×1 Lattice on Sb (111). 2D Mater. 2016, 3, 045005.

(10) Zhu, F. F.; Chen, W. J.; Xu, Y.; Gao, C. L.; Guan, D. D.; Liu, C. H.; Qian, D.; Zhang, S. C.; Jia, J. F. Epitaxial Growth of Two-Dimensional Stanene. Nat. Mater. 2015, 14, 1020-1025.

(11) Gou, J.; Kong, L.; Li, H.; Zhong, Q.; Li, W.; Cheng, P.; Chen, L.; Wu, K. Strain-Induced Band Engineering in Monolayer Stanene on Sb(111). Phys. Rev. Mater. 2017, 1, 54004.

(12) Chiappe, D.; Scalise, E.; Cinquanta, E.; Grazianetti, C.; Van Den Broek, B.; Fanciulli, M.; Houssa, M.; Molle, A. Two-Dimensional Si Nanosheets with Local Hexagonal Structure on a MoS2 Surface. Adv. Mater. 2014, 26, 2096-2101.

(13) Zhang, L.; Bampoulis, P.; Rudenko, A. N.; Yao, Q.; Van Houselt, A.; Poelsema, B.; Katsnelson, M. I.; Zandvliet, H. J. W. Structural and Electronic Properties of Germanene on MoS2. Phys. Rev. Lett. 2016, 116, 256804.

(14) D’Acapito, F.; Torrengo, S.; Xenogiannopoulou, E.; Tsipas, P.; Marquez Velasco, J.; Tsoutsou, D.; Dimoulas, A. Evidence for Germanene Growth on Epitaxial Hexagonal (h)-AlN on Ag(111). J. Phys. Condens. Matter 2016, 28, 045002R. 
(15) Van Bremen, R.; Yao, Q.; Banerjee, S.; Cakir, D.; Oncel, N.; Zandvliet, H. J. W.; Intercalation of Si between MoS2 Layers. Beilstein J. Nanotechnol. 2017, 8, 1952-1960.

(16) Wang, W.; Uhrberg, R. I. G. Investigation of the Atomic and Electronic Structures of Highly Ordered Two-Dimensional Germanium on Au(111). Phys. Rev. Mater. 2017, 1, 74002.

(17) Nath, K. G.; Shimoyama, I.; Sekiguchi, T.; Baba, Y. Chemical-State Analysis for LowDimensional Si and Ge Films on Graphite. J. Appl. Phys. 2003, 94, 4583-4588.

(18) De Crescenzi, M.; Berbezier, I.; Scarselli, M.; Castrucci, P.; Abbarchi, M.; Ronda, A.; Jardali, F.; Park, J.; Vach, H. Formation of Silicene Nanosheets on Graphite. ACS Nano. 2016, 10, 1116311171.

(19) Persichetti, L.; Jardali, F.; Vach, H.; Sgarlata, A.; Berbezier, I.; Crescenzi, M. De; Balzarotti, A. Van Der Waals Heteroepitaxy of Germanene Islands on Graphite. J. Phys. Chem. Lett. 2016, 7, 3246-3251.

(20) Marsen, B.; Lonfat, M.; Scheier, P.; Sattler, K. Energy Gap of Silicon Clusters Studied by Scanning Tunneling Spectroscopy. Phys. Rev. B. 2000, 62, 6892-6895.

(21) Kushvaha, S. S.; Yan, Z.; Xiao, W.; Xu, M. J.; Xue, Q. K.; Wang, X. S. Self-Assembled Ge, Sb and Al Nanostructures on Graphite: Comparative STM Studies. Nanotechnology 2007, 18, 145501.

(22) Nezhdanov, A. V.; Filatov, D. O.; Antonov, D. A.; Zubkov, S. Y.; Mashin, A. I.; Ershov, A. V. The Morphology, Electron Structure, and Optical Properties of Self-Assembled Silicon Nanostructures on the Surface of Highly Oriented Pyrolytic Graphite. Semiconductors 2011, 45, 5660.

(23) Shimonaka, M.; Nakamura, Y.; Kikkawa, J.; Sakai, A. Self-Assembly of Ge Clusters on Highly Oriented Pyrolytic Graphite Surfaces. Surf. Sci. 2014, 628, 82-85.

(24) Prévot, G.; Bernard, R.; Cruguel, H.; Curcella, A.; Lazzeri, M.; Leoni, T.; Masson, L.; Ranguis, A.; Borensztein, Y. Formation of Silicene on Silver: Strong Interaction between Ag and Si. Phys. Status Solidi B 2016, 253, 206-217.

(25) Díaz Álvarez, A.; Zhu, T.; Nys, J. P.; Berthe, M.; Empis, M.; Schreiber, J.; Grandidier, B.; Xu, T. Scanning Tunnelling Spectroscopy and Raman Spectroscopy of Monolayer Silicene on $\operatorname{Ag}(111)$. Surf. Sci. 2016, 653, 92-96.

(26) Giunta, P. L.; Kelty, S. P. Direct Observation of Graphite Layer Edge States by Scanning Tunneling Microscopy. J. Chem. Phys. 2001, 114, 1807-1812.

(27) Niimi, Y.; Matsui, T.; Kambara, H.; Tagami, K.; Tsukada, M.; Fukuyama, H. Scanning Tunneling Microscopy and Spectroscopy of the Electronic Local Density of States of Graphite Surfaces near Monoatomic Step Edges. Phys. Rev. B. 2006, 73, 085421

(28) Cai, Y.; Chuu, C. P.; Wei, C. M.; Chou, M. Y. Stability and Electronic Properties of TwoDimensional Silicene and Germanene on Graphene. Phys. Rev. B. 2013, 88, 245408.

(29) Tománek, D. ; Louie, S.G. First-Principles Calculation of Highly Asymmetric Structure in Scanning Tunneling Microscopy Images of Graphite. Phys. Rev. B. 1988, 37, 8327. 
(30) Li, G.; Luican, A.; Andrei, E. Y. Scanning Tunneling Spectroscopy of Graphene on Graphite. Phys. Rev. Lett. 2009, 102, 176804.

(31) Stephan, R.; Derivaz, M.; Hanf, M. C.; Dentel, D.; Massara, N.; Mehdaoui, A.; Sonnet, P.; Pirri, C. Tip-Induced Switch of Germanene Atomic Structure. J. Phys. Chem. Lett. 2017, 8, 45874593.

(32) Mizes, H. A.; Foster, J. S. Long-Range Electronic Perturbations Caused by Defects Using Scanning Tunneling Microscopy. Science 1989, 244, 559-562.

(33) Shedd, G. M.; Russell, P. E. A Simple Wave Function Interference Model for the ElectronDensity Superstructures Observed during Scanning Tunneling Microscopy of Perturbed Graphite Surfaces. Surf. Sci. 1992, 266, 259-264.

(34) Takeuchi, N.; ValenzuelaBenavides, J.; delaGarza, L. M. Electronic Superstructures on the Graphite Surface Studied by First-Principles Calculations. Surf. Sci. 1997, 380, 190-198.

(35) Ruffieux, P.; Grüning, O.; Schwaller, P.; Schlapbach, L.; Grüning, P. Hydrogen Atoms Cause Long-Range Electronic Effects on Graphite. Phys. Rev. Lett. 2000, 84, 4910-4913.

(36) Xhie, J.; Sattler, K.; M̈ller, U.; Venkateswaran, N.; Raina, G. Periodic Charge-Density Modulations on Graphite near Platinum Particles. Phys. Rev. B. 1991, 43, 8917-8923.

(37) Kondo, T.; Iwasaki, Y.; Honma, Y.; Takagi, Y.; Okada, S.; Nakamura, J. Formation of Nonbonding $\pi$ Electronic States of Graphite due to Pt-C Hybridization. Phys. Rev. B. 2009, 80, 233408 .

(38) Xu, H.; Permana, H.; Lu, Y.; Ng, K. Y. S. STM Study of Mo Growth and Induced Surface Structure Changes on HOPG. Surf. Sci. 1995, 325, 285-293.

(39) Francis, G. M.; Goldby, I. M.; Kuipers, L.; von Issendorf, B.; Palmer, R. E. Deposition and Growth of Noble Metal Clusters on Graphite. J. Chem. Soc. Dalt. Trans. 1996, 5, 665.

(40) Bai, A.; Ukuyama, S.; Yokogawa, K.; Yoshimura, M. Characteristics of the $(\sqrt{3} 3 \sqrt{ } 3) R 30^{\circ}$ Superstructure of Graphite by Scanning Tunneling Microscopy. Jpn. J. Appl. Phys. 2000, 39, 43474350.

(41) Tapasztó, L.; Nemes-Incze, P.; Osváth, Z.; Bein, M. C.; Darabont, A.; Biró, L. P. Complex Superstructure Patterns near Defect Sites of Carbon Nanotubes and Graphite. Phys. E Low-Dimens. Syst. Nanostruct. 2008, 40, 2263-2267.

(42) López, J. C. M.; Passeggi, M. C. G.; Ferrón, J. Surface Superstructures in Highly Oriented Pyrolytic Graphite Surfaces after Ar+ Bombardment. Surf. Sci. 2008, 602, 671-676.

(43) Mizes, H. A.; Park, S. I.; Harrison, W. A. Multiple-Tip Interpretation of Anomalous ScanningTunneling-Microscopy Images of Layered Materials. Phys. Rev. B. 1987, 36, 4491-4494. 